\title{
Adequate Theory of Oscillator: A Prelude to Verification of Classical Mechanics Part 2
}

\author{
Zdzisław Pluta, Tadeusz Hryniewicz* \\ Faculty of Mechanical Engineering, Koszalin University of Technology, \\ Racławicka 15-17, 75-620 Koszalin, Poland \\ *E-mail address: Tadeusz.Hryniewicz@tu.koszalin.pl
}

"The essence of scientific discovery relies on the fact that one looks at the same what everyone sees and notices what nobody has seen."

L. Pauling

\begin{abstract}
In the paper, the adequate theory of oscillator is presented, being a sort of prelude to verification of the classical theory of mechanics. In the first part of the paper the principle of energy conservation was considered. This second part of the paper is to present the energetic aspects of oscillator motion. Here also an exemplary real system corresponding with the existent differential equation of oscillator is given. They are to develop the kinetics of a body in the harmonic motion, to be presented in the next Part of the work.
\end{abstract}

\section{Keywords}

Oscillator; Energy; Principle of energy conservation; Space-time; Potential field; Gravitation

\section{INTRODUCTION}

Part 1 of the paper served to introduce readers into the problems of a real necessity for verification of the classical mechanics [1]. General characteristics of the classical mechanics were presented based on the references. Exemplification of artifacts of classical theory of oscillator was developed. Part 2 of the paper is to consider the energetic aspects of oscillator motion, by description of an exemplary real system motion in reference to the existent differential equation of oscillator motion. 


\section{ENERGETIC ASPECTS OF OSCILLATOR MOTION}

There were lots of papers and texts describing the essence of energy. The characteristic feature is that different meanings are assigned to it, and sometimes even enigmatic ones. There is still no univocal meaning in science as to the definition of energy, with this also to its essence. The classical physics has assumed continuity in energy transformation/change, saying de facto about the work, whereas a quantum physics represents a reverse approach concerning the character of changes of this magnitude. According to the quantum physics, the energy changes discontinuously, but on a jump way.

The works [2, 3] and the book [4] cover a broad explanation of the essence of energy. Undoubtedly the energy changes by a jump way but the sum of all energies of a system is unchanged, changing by the jump way only into the site of its concentration. One should add that the last feature covers also the gravitation energy itself.

For the need of this work, there will be only the new knowledge elements used (not existent in the daylight as yet) which will satisfactorily allow to explain energetic nature of the oscillator motion. All definitions and deep philosophical considerations as well as considerably developed critical analysis of the existent state of knowledge treating about the energy as essentially of total natural reality, are to be omitted.

Here is the considered oscillator (Fig. 4), being the system in the form of a pull spring of the coefficient of elasticity $k$ with a body of mass $m$ fixed to it. In the upper position of the system, at the height of a transitory unstable static potential field (USPF) ${ }^{\bullet}$, the unstable energetic balance of this mass-elastic system takes place. That means no force is contained in itself or the forces do not co-exist one with each other but are clearly separated. Thus the balance exists between the forces $F_{o}, Q, B_{o}$, with the following form: $F_{o}=Q+B_{o}$. The particular symbols denote the forces of: holding (that may be a biologic force, with a holder being simply a palm of the hand), gravity, and inertia, respectively. The described potential field is zero or the initial field, so its denotation is $\mathrm{O}-\mathrm{O}$.

When a body is displaced down, at first moment it will be at momentary unstable static potential field (TUPF) ${ }^{\circ}$, where interaction of a spring is noticed, determined here by the initial elasticity force $S_{o}$.

In the next step the body passes through the space-time (dotted area in Fig. 4), and all forces change linearly (expressed as the function of way length) according to the scheme presented in the lower part of Fig. 4. After passing the body through the space-time of the length $x_{s}$, referred to the static elongation of a spring, the body approaches temporary stable and static potential field (SSPF) ${ }^{\bullet}$. Positions of these fields (instantaneous and temporary) are surely equal with some change of their role only.

On the instantaneous field, there is still an interaction of palm, determined by the force $F_{l}$, and then there were only remaining forces, such as $Q, B_{l}, S_{l}$. A significant thing, the gravity force co-exists here with the inertia force that can be written as $Q \otimes B_{1}$. This in turn corresponds to two following identities: $Q=S_{1}, B_{1}=S_{l}$. A new second position of the body, corresponding to the neighboring potential field, has been denoted additionally by a symbol 1 -1 . 


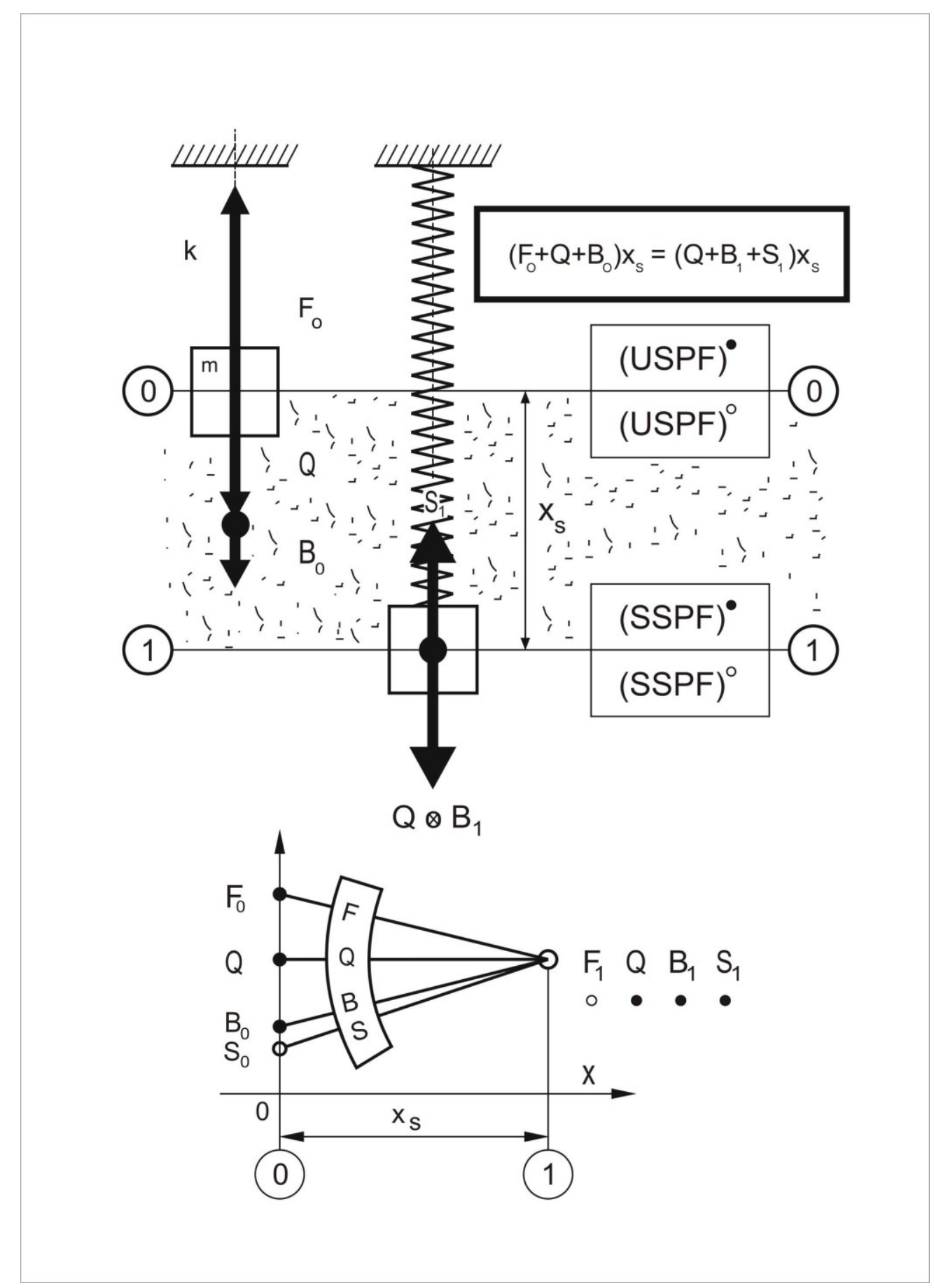

Fig. 4. Harmonic oscillator in unstable static potential fields

Intervals and courses of variability of all forces which determine the mutual interaction of the system elements are contained in Table 1. Wheels here denote a temporary potential field and the circles correspond with the instantaneous fields of this type. 
Table 1. Intervals and courses of forces variability determining mutual interaction of the system elements of all bodies

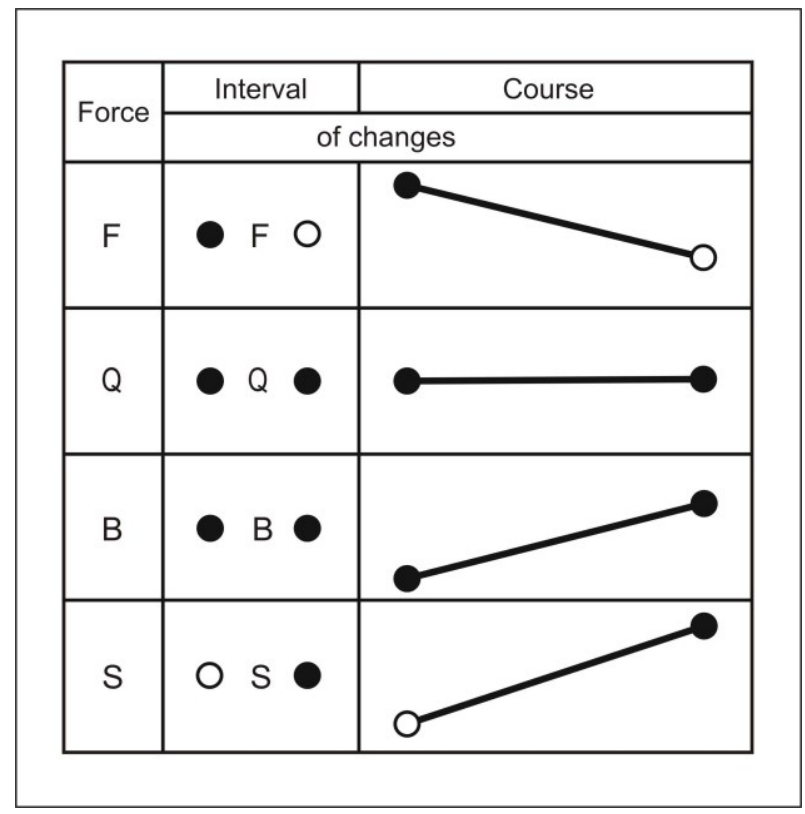

Even if the static balance on both neighboring fields was different quantitatively, the energetic balance is analogous. It cannot be different because this is said by a properly understood principle of energy conservation, or more exactly the principle of conservation of their measures, or potentials [4]. Thus the energy is not a physical but rather mental magnitude. This is why a measure is needed with the potential being the measure.

The principle of energy measure conservation of the analyzed system possesses the following general form:

$$
V_{F}^{(0)}+V_{Q}^{(0)}+V_{B}^{(0)}=V_{Q}^{(1)}+V_{B}^{(1)}+V_{S}^{(1)}
$$

where the following potentials are: $V_{F}$ - biologic, $V_{Q}$ - gravitational, $V_{B}$-inertia, $V_{S}$ - elastic; with the marks in parentheses at the place of powers meaning numbers of potential fields.

A detailed form of this principle possesses this configuration (denoted also in Figure 4):

$$
\left(F_{0}+Q+B_{0}\right) x_{s}=\left(Q+B_{1}+S_{1}\right) x_{s}
$$

That means the following

$$
F_{0}+Q+B_{0}=3 Q
$$

because $B_{1}=S_{1}=Q$.

Taking into account the earlier mentioned equilibrium of forces on the initial potential field, that is:

$$
F_{0}=Q+B_{0}
$$

one obtains two linear equations, namely (5) and (6). Their solution, due to $B_{o}$ and $F_{o}$, leads to the result: 


$$
B_{0}=\frac{Q}{2}, \quad F_{0}=\frac{3}{2} Q
$$

One may, of course, without any difficulty describe courses of all forces and then particular works in the interstate space. This, however, will be omitted, firstly because of the simplicity of that partial problem, secondly due to the limited frames of this paper.

It is time now for energetic description of the free motion of oscillator. Next scheme serves to explain it (Fig. 5). In this case only instantaneous potential fields should be taken into account. At the beginning, the energetic temporary equilibrium of the body on two consecutive potential fields will be described. It is important first to determine the length of second and further space-times which, as it is known, for the harmonic motion will be equal.

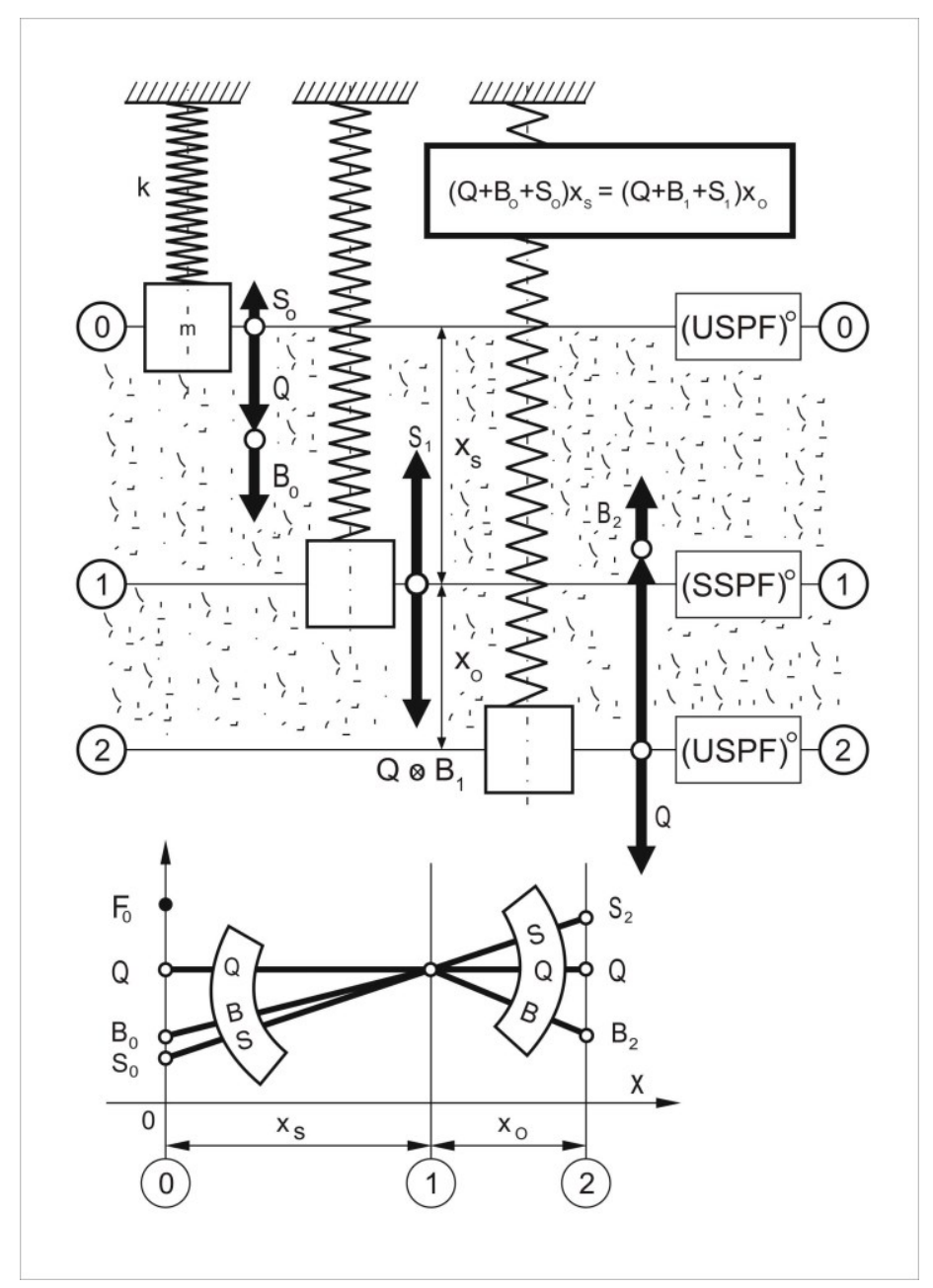

Fig. 5. Scheme of energetic positions of oscillator in its free motion

The principle of conservation of energy measures for a free motion, which takes place here, is presented as

$$
V_{Q}^{(0)}+V_{B}^{(0)}+V_{S}^{(0)}=V_{Q}^{(1)}+V_{B}^{(1)}+V_{S}^{(1)}
$$


and its detailed form possesses the structure:

$$
\left(Q+B_{0}+S_{0}\right) x_{s}=\left(Q+B_{1}+S_{1}\right) x_{0}
$$

Assuming $B_{1}=S_{1}=Q$, and $B_{o}=Q / 2$, one obtains:

$$
\left(\frac{3}{2} Q+S_{0}\right) x_{s}=3 Q x_{0}
$$

with

$$
x_{0}=\frac{\frac{3}{2} Q+S_{0}}{3 Q} x_{s}
$$

It is worth noting that the inertia force is always directed into the stable position of the system, that is a stable static potential field (SSPF) ${ }^{\circ}$. Such is the nature of inertia, of the real inertia force [4]. When considering a reverse sense to the sense of velocity vector one assumes a fictitious world, created, as it is known, by d'Alembert and then developed mathematically by the creativity of Lagrange and Hamilton.

The principle of conservation of energy measures (potentials) for each consecutive potential field is created by analogy, as the product of forces (intensities of particular fields) and the length of space-time through which the considered material body is to penetrate. It should be added that for the even fields $(2,4,6, \ldots)$ which are instantaneous unstable static potential fields (USPF) ${ }^{\circ}$ corresponding to upper $(+)$ and lower position of the body $(-)$ one should additionally take into account existent there the internal energies $\mathrm{U}^{+}, \mathrm{U}^{-}$, respectively (Fig. 6).

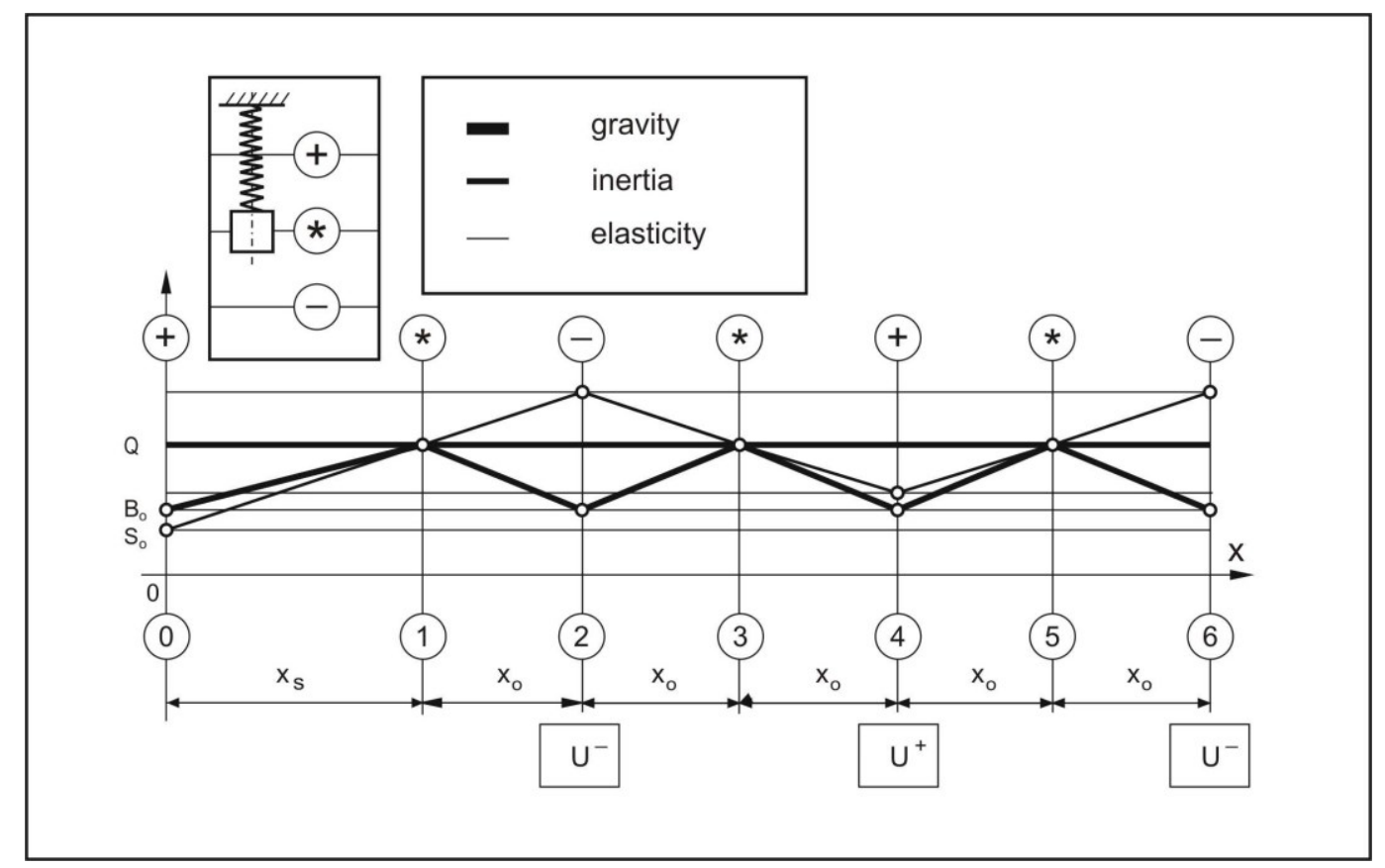

Fig. 6. Courses of forces for freely vibrating mass-elastic system and the position of instantaneous potential fields 
For example, for the $2^{\text {nd }}$ field this principle of conservation takes the following form:

$$
V_{S}^{(2)}+V_{Q}^{(2)}+V_{B}^{(2)}+U^{-}=i d e m
$$

For the following even field, the $4^{\text {th }}$ one, this principle of conservation takes the form:

$$
V_{S}^{(4)}+V_{Q}^{(4)}+V_{B}^{(4)}+U^{+}=i d e m
$$

Moreover one should notice that the internal energy of the system in the upper position will be always higher than such an energy in the lower position.

\section{DESCRIPTION OF MOTION OF AN EXEMPLARY REAL SYSTEM CORRESPONDING TO EXISTENT DIFFERENTIAL EQUATION OF OSCILLATOR MOTION}

In the free motion, only in a stable potential field there is a force equilibrium lasting just a while for a moment of the body transition to the neighboring time-space.

Nowhere there is such an equilibrium and it cannot be. D'Alembert obtained though an equilibrium but by fictitious way/approach by introducing to the system so called fictitious inertia force.

That, however, disqualifies/condemns this speculative theory based on such mathematical treatment of reality. The mechanism of such erroneous procedure was explained earlier in Part 1 (Chapter 3) concerned with exemplification of artifacts of the classical theory of oscillator.

The identical amplitude of harmonic motion, commencing from the lower position of the body as well as its constant vibration period, does not present the proof that the motion may be described by a sinusoid equation.

Though in the space-times, between the potential fields, at the same time the body may be displaced on other way (do not mix it with a path or trajectory). That approach is to be explained further on.

Firstly, however, an example of a real system has been presented of which the kinematics allows to obtain motion of a working element in accordance to a sinusoidal course.

It is just a crank-sinusoidal mechanism (Fig. 7) with a link/slider displacing with such a motion. It has been firmly connected with a guide/slideway and a crank connected by the slider rotates with a constant angle velocity. 


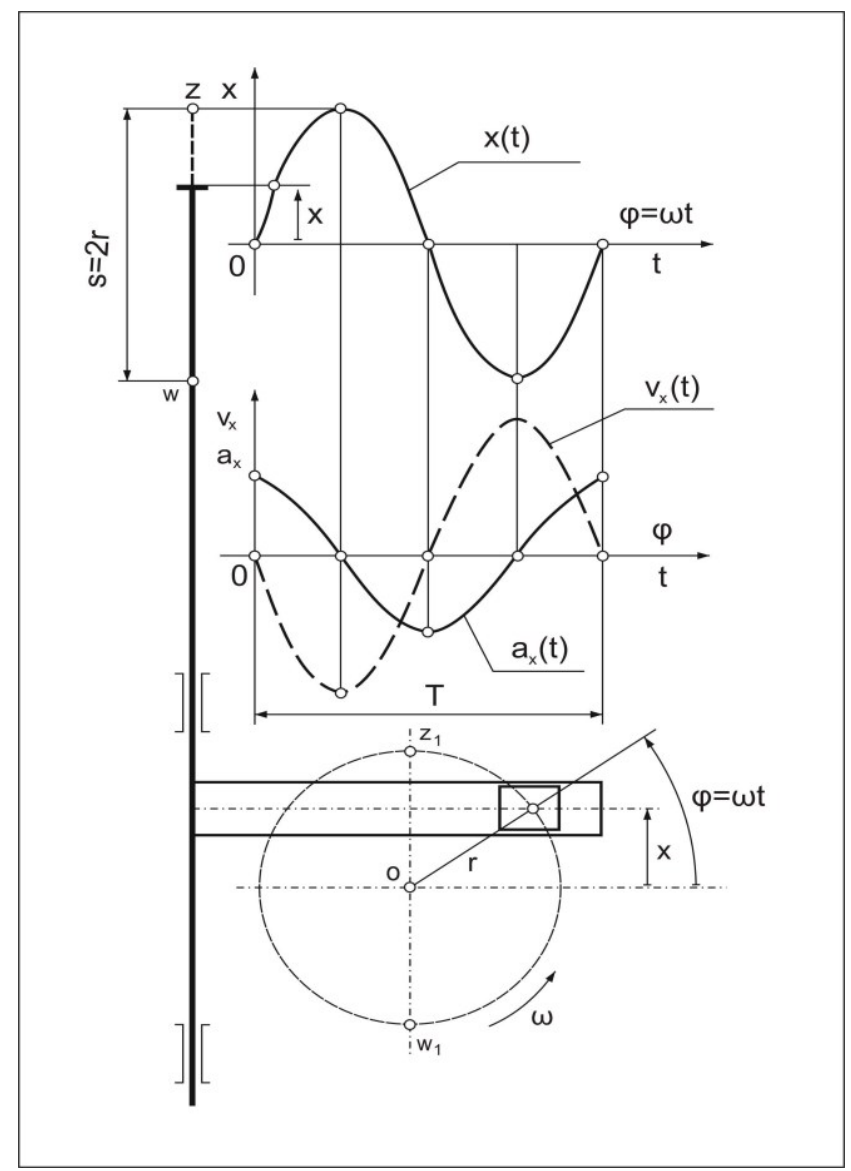

Fig. 7. Crank-sinusoidal mechanism

Returning to the d'Alembert's oscillator motion description one should notice that it presents only changes of the elasticity force and the inertia force as:

$$
m \ddot{x}+k x=0
$$

with a force of gravity, as apparent, removed by a mathematic way. A deep reflection of this subject has seen its daylight in $[5,6]$.

In the reality there is a material system which may be described by this equation (14), however, the site of fictitious inertia force will be taken by other force being a measure of determined real behaviour of a movable system.

This description will be concerned unfortunately only on the changes of determined coordinate of the body position of a rotating system. A variable motion, of rather its shadow than the body, will be described.

Let a plate in the form of disk be a material system (Fig. 8). On the plate there is a block affixed to a pull spring which in turn is fastened into the disk axis. The block possesses mass $m$ and the spring is characterized by a coefficient of elasticity $k$. 


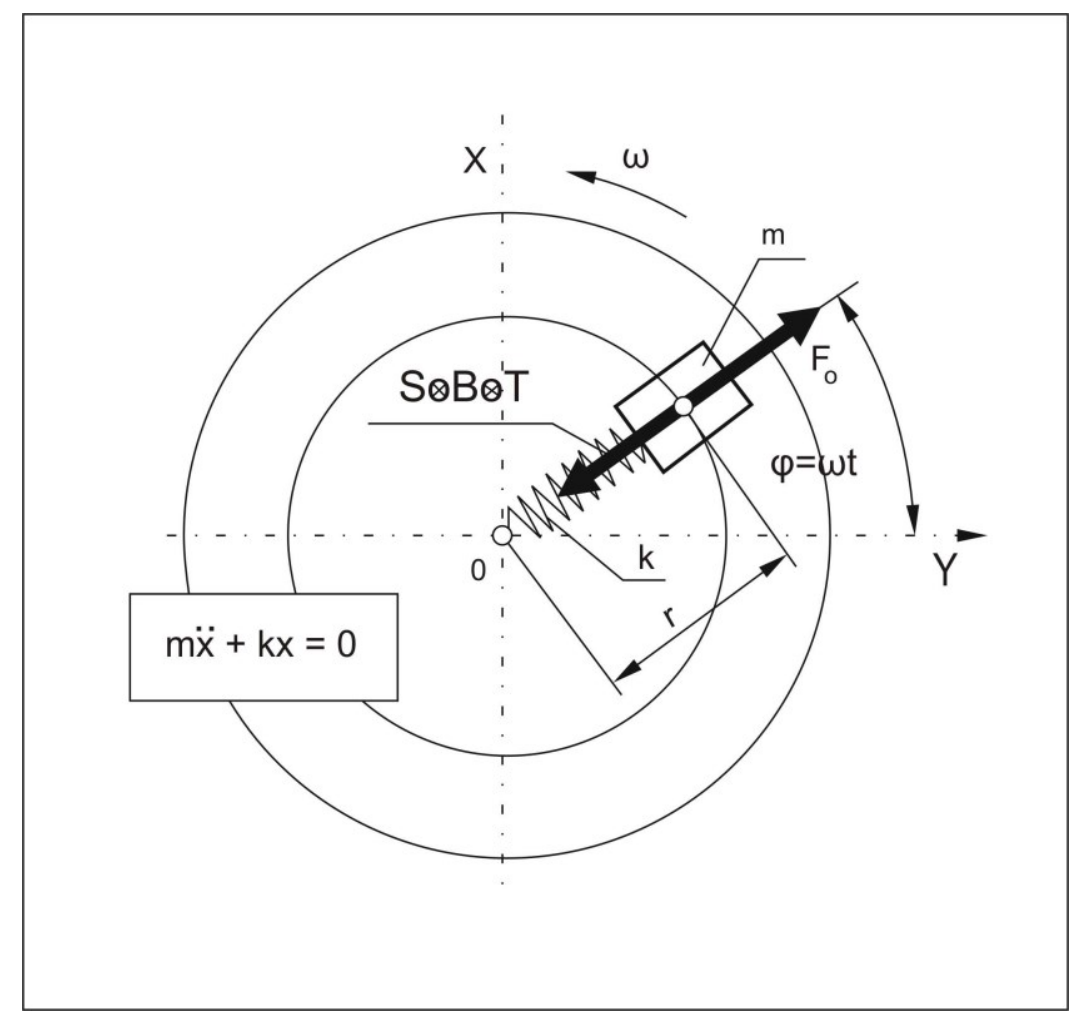

Fig. 8. Rotating mass-spring system being in stable state

The whole system is in a stable state. That means the inertia force $B$ is the biggest one and co-exists with the forces of: elasticity $S$, and surface resistance $T$. It can be written as follows: $S \otimes B \otimes T$. It is a triple co-existence (one could say: three in one). The system rotates with a constant angle velocity $\omega$ and a centrifugal inertia is denoted with the measure being the centrifugal inertia force $F_{o}$.

A scalar equilibrium of forces in the direction 0 X, i.e. $F_{o x}=S_{x}$, has the following form:

$$
F_{0} \sin \omega t=S \sin \omega t
$$

expressed in the function of time $t$ connected with the angular displacement $\varphi$ by a known relationship $\varphi=\omega t$.

Taking into account that $F_{o}=m \omega^{2} r$, and $S=k r$, one obtains:

with

$$
m \omega^{2} r \sin \omega t=k r \sin \omega t
$$

$$
-m \ddot{x}=k x
$$

because $x=r \sin \omega t, \quad \ddot{x}=-r \omega^{2} \sin \omega t$. Of course further on, after locating all terms on one side of the equation, one may finely obtain the commonly known equation (14) with one exception, that it possesses a physical meaning though it still simulates the oscillatory motion. 


\section{CONCLUSION}

These Chapters do not conclude the paper, but are rather the second step to the interstate before a passage to the real problems of existent classical mechanics. Next two parts of the paper will develop the kinetics of a body in the harmonic motion as the introduction to verification of the adequate theory of oscillator. Then the gravity acceleration will be determined by means of two independent methods.

\section{REFERENCES}

[1] Zdzisław Pluta, Tadeusz Hryniewicz, International Letters of Chemistry, Physics and Astronomy 2 (2012) 28-34

[2] Zdzisław Pluta, LAB 4 (2005) 43-46

[3] Zdzisław Pluta, Energetyka 7 (2005) 496-501 (in Polish)

[4] Zdzisław Pluta, Fundamentals of surface smoothing by elastic wheels, Monograph of Politechnika Koszalińska, Koszalin 2007, (in Polish)

[5] Zdzisław Pluta, LAB 2 (2004) 50-52

[6] Zdzisław Pluta, LAB 6 (2004) 42-46 